\title{
Kosten und Erlöse bei der Co-Vergärung von mechanisch aufbereitetem Bioabfall in Faultürmen kommunaler Kläranlagen
}

\author{
Anna Jank · Christian Ebner · Wolfgang Müller · Christian Fimml · Rudolf Markt · Anke Bockreis
}

Online publiziert: 28. Juni 2017

(c) Der/die Autor(en) 2017. Dieser Artikel ist eine Open-Access-Publikation.

\begin{abstract}
Zusammenfassung Die moderne Abwasserbehandlung weist ein großes Potenzial zur Bereitstellung erneuerbarer Energien auf. Auf Kläranlagen kann die chemische Energie im Abwasser genutzt werden, indem in den Faultürmen Biomethan produziert wird. Diese Biomethanproduktion kann durch die gezielte Zugabe organischer Abfälle in die Faultürme gesteigert werden (= Co-Vergärung). Abfälle, die in den Faulturm als Co-Substrate zugegeben werden, müssen pumpfähig und in den Faulschlamm einmischbar sein. Typische Abfallströme, die dafür in Frage kommen sind z. B. Speisereste aus Großküchen oder Molkereiabfälle. Derartige Abfälle können meist nach geringer mechanischer Aufbereitung dem Faulturm beigemischt werden. Diese Abfälle sind begehrte Reststoffe der Kläranlage, da sie im Allgemeinen weitgehend frei von Störstoffen wie Glas, Steinen oder Kunststoffen sind.
\end{abstract}

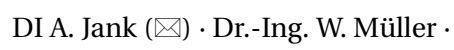
Univ.-Prof. Dr.-Ing. A. Bockreis Institut für Infrastruktur, Arbeitsbereich Umwelttechnik, Fachgebiet Abfallbehandlung und Ressourcenmanagement, Universität Innsbruck,

Technikerstraße 13, 6020 Innsbruck, Österreich

anna.jank@uibk.ac.at

DI A. Jank · Dr. C. Ebner alpS $\mathrm{GmbH}$,

Grabenweg 68, 6020 Innsbruck, Österreich

\section{Fimml}

Abwasserverband

Achental-Inntal-Zillertal (AIZ), Strass im Zillertal 150, 6261 Strass im Zillertal, Österreich

\section{R. Markt}

Institut für Mikrobiologie, Universität Innsbruck, Technikerstraße 25d, 6020 Innsbruck, Österreich
Die begrenzte Verfügbarkeit von Speiseresten oder Molkereiabfällen führt zunehmend dazu, dass auch Bioabfälle aus der Getrenntsammlung oder überlagerte Lebensmittel mechanisch aufbereitet und in den Kläranlagen mitverarbeitet werden. Bei der Nutzung derartiger Substrate ist vermehrt mit Folgeproblemen auf der Kläranlage zu rechnen, da sie stärker mit Störstoffen belastet sind. Durch Abrasion an Aggregaten, verlegte Leitungen oder Sedimenten im Faulturm ergeben sich Mehrkosten für die Kläranlage. Bei der Betrachtung der Mehrkosten durch die Co-Vergärung von mechanisch aufbereitetem Bioabfall müssen neben den Aufwendungen, die auf Störstoffe zurückzuführen sind, auch Kosten für den Schlammanfall aus den Co-Substraten und der Rückbelastung, insbesondere von Stickstoff, in die Abwasserreinigung der Kläranlage berücksichtigt werden. In dieser Arbeit wird eine ökonomische Betrachtung aufgestellt, die die relevanten Kosten der Co-Vergärung erfasst und den Erlösen aus der Verwertung des zusätzlich produzierten Biomethans gegenüberstellt.

\section{Costs and revenues for co- digestion of mechanically pretreated biowaste in wastewater treatment plants}

Abstract The production of methane in wastewater treatment plants can be increased by applying co-digestion. Leftovers from canteens or waste from dairy production are frequently used for co-digestion in wastewater treatment plants. These substrates are free from impurities and pumpable and hence almost no further mechanical treatment is necessary before adding these wastes to the fermentation process. Due to the limited availability of suitable leftovers etc., also separately collected municipal biowastes or packed food wastes from supermarkets are used as co-sub- strates. These waste materials need to be milled or shredded before digestion. Although impurities like plastic films are separated, other impurities, such as stones and glass particles, can reach the digester. These impurities are a cause for extra costs for the wastewater treatment plant operators because they can provoke plant malfunctions, increased equipment wear, pipe clogging, or accumulation of sediments in the digester. These costs caused by impurities in the pretreated biowaste are investigated in this work. Furthermore, the costs for the ensuing additional sludge disposal and additionally required removal of nitrogen because of added co-substrates are considered. All additional costs caused by the co-digestion of biowaste in wastewater treatment plants are compiled and compared with the revenue generated by the additionally produced methane from biowaste (production of electrical power for use at the wastewater treatment plant, selling the produced energy or selling the methane).

\section{Einleitung}

Innerhalb der kommunalen Infrastruktur stellen Kläranlagen einen der bedeutendsten Energieverbraucher dar. Gleichzeitig aber weist die moderne Abwasserbehandlung auch ein großes Potenzial für die Bereitstellung erneuerbarer Energien auf. Auf Kläranlagen kann die chemische Energie im Abwasser genutzt werden, indem in den Faultürmen Biomethan produziert wird. Co-Vergärung ist die gleichzeitige Vergärung von mindestens zwei unterschiedlichen Substraten und eine Option, den Gasertrag der Vergärung zu steigern. Meistens sind Primär- und Überschussschlamm die Hauptsubstrate an den Kläranlagen, denen als CoSubstrat organische Reststoffe zugegeben werden. Die Mehrenergie, die dabei produziert wird, kann direkt von der 
Kläranlage zur Abdeckung des Eigenstrombedarfs genutzt werden, wodurch ein energieautonomer Kläranlagenbetrieb ermöglicht werden kann (Ebner 2013). Je nach Standortgegebenheiten kann der produzierte Strom bzw. das produzierte Methan auch in das Strombzw. Gasnetz eingespeist werden.

Die Rahmenbedingungen für eine Verwertung von organischen Abfällen an einer Kläranlage sind günstig. Faulraumkapazitäten sind vielfach vorhanden, da diese durch die alleinige Vergärung der Abwasserschlämme häufig nicht voll ausgelastet werden (Krupp et al. 2005; Montusiewicz and Lebiocka 2011). Werden organische Abfälle auf Kläranlagen mitvergoren, kann bereits bestehende und für die Schlammbehandlung notwendige Infrastruktur mitgenutzt und somit optimal ausgelastet werden. In der Regel müssen nur einige wenige Anlagenteile wie Substratbunker, Leitungen und Pumpen für die Zudosierung der Co-Substrate neu errichtet werden. Die Investitionskosten sind somit im Vergleich zu Anlagen für die Monovergärung von Bioabfällen deutlich reduziert. Kläranlagen verfügen über fachlich geschultes Personal inkl. Bereitschaftsdienst, die für die beschriebenen Aufgaben bestens qualifiziert sind. Somit erleichtert geschultes Kläranlagenpersonal, vorhandene Kläranlageninfrastruktur wie Leitungen oder Pumpen bis hin zu freien BHKW-Kapazitäten zur Stromerzeugung, die Umsetzung der Co-Vergärung auf der Kläranlage. Der Faulschlamm, in den der organische Abfall zugegeben wird, ist meist gekennzeichnet durch ein enges $\mathrm{C} / \mathrm{N}$ Verhältnis und eine hohe Pufferkapazität (Iacovidou et al. 2012; Silvestre et al. 2011). Dadurch ist eine Co-Vergärung von Substraten mit hohen Anteilen an leicht abbaubarer Organik und niedrigem $\mathrm{pH}$-Wert meist unbedenklich (Dai et al. 2013; Kim et al. 2011).

Durch eine rasche Selbstversäuerung organischer Abfälle werden diese gewissermaßen konserviert (Aichinger et al. 2015; Schneider et al. 2015). Dadurch ist es möglich, dieses Material in Speicherbehältern an der Kläranlage zu lagern, ohne dass dies negative Auswirkungen, wie eine Verminderung der Energiedichte des Substrats hat. Je nach Erfordernis kann Substrat in den Faulturm zudosiert und aus dem gebildeten Methan Strom bedarfsgerecht produziert werden.
Pumpfähige Abfallströme, die in den Faulschlamm einmischbar sind, kommen als Substrate für die Co-Vergärung in Frage. Saubere, störstofffreie Abfälle, die ohne weitere Aufbereitung vergärt werden können, sind von den Anlagen begehrte Reststoffe (Lastella et al. 2002). Molkereiabfälle oder Speisereste entsprechen im Wesentlichen diesen Anforderungen. Da diese jedoch nur begrenzt verfügbar sind, werden zunehmend andere Stoffe wie Bioabfall aus der Getrenntsammlung oder überlagerte Lebensmittel als Substratquellen für die Co-Vergärung verwendet. Solche Abfälle müssen vor der Vergärung an der Kläranlage mechanisch aufbereitet werden. Dabei wird der Abfall häufig zerkleinert und durch entsprechende Wasserzugabe pumpfähig gemacht. Nach der Aufbereitung von überlagerten Lebensmitteln bzw. Bioabfällen sind im Substrat jedoch immer auch Störstoffe wie Glas, Sand, Steine oder auch Aluminium enthalten, bedingt durch Fehlwürfe bei der Sammlung bzw. durch die mechanische Aufbereitung, die Glas, Steine oder Sand kaum abtrennen kann. Werden solche Störstoffe in den Vergärungsprozess eingeschleust, kann dies zu erhöhtem Reparaturaufwand an Anlagenteilen der Kläranlage, zu Verstopfungen in Rohrleitungen oder $\mathrm{zu}$ Ablagerungen im Faulturm führen (Bayo et al. 2015; Novarino und Zanetti 2012; RomeroGüiza et al. 2014; Shen et al. 2015).

Darüber hinaus fallen bei einer CoVergärung organischer Reststoffe zusätzliche Kosten durch eine intensivere Ausnützung des Faulturms bzw. der Kläranlageninfrastruktur an.

\subsection{Ziel dieser Arbeit}

Die Co-Vergärung organischer Reststoffe, wie getrennt gesammelte Bioabfälle oder überlagerte Lebensmittel nach einer mechanischen Aufbereitung, im Folgenden als mechanisch aufbereiteter Bioabfall (MAB) bezeichnet, wird seit einigen Jahren in Tirol in stetig wachsendem Umfang betrieben. In dieser Zeit wurden zum einen viele praktische Erfahrungen an den Anlagen selbst, zum anderen auch Ergebnisse im Rahmen von Forschungsprojekten erarbeitet. In der vorliegenden Arbeit sollen diese Erfahrungen bzw. Informationen in einer gesamthaften, ökonomischen Betrachtung zusammengefasst werden. Auf der Kostenseite werden alle relevanten Kosten erfasst, welche durch die Verarbeitung von MAB an den Kläranlagen entstehen. Auf der Erlösseite werden verschiedene Verwertungsmöglichkeiten des zusätzlich produzierten Biomethans mit den aktuellen Marktpreisen wirtschaftlich bewertet.

\section{Untersuchungsrahmen}

\subsection{Betrachtete Kläranlagen}

Für die vorliegende Arbeit wurden Betriebsdaten von zwei Tiroler Kläranlagen ausgewertet, die beide seit mehreren Jahren $\mathrm{MAB}$ in größerem Umfang co-vergären.

Die ARA Strass liegt in Tirol am Eingang des Zillertals und ist für die Abwasserreinigung für 32 Verbandsgemeinden verantwortlich. Die Anlage wurde 1989 in Betrieb genommen. Die Ausbaugröße der Anlage beträgt $167.000 \mathrm{EW}_{60}$. Es handelt sich um eine zweistufige biologische Kläranlage nach dem A-B-Prinzip. Eine Besonderheit der Anlage ist die Filtratwasserbehandlung nach dem DEMON ${ }^{\circledR}$-Verfahren, welche 2004 in Betrieb genommen wurde. Im Jahr 2008 wurde an der ARA Strass mit der Co-Fermentation von MAB begonnen (in den Jahren davor wurden schon Fettabscheiderinhalte und Molkereiabfälle mitvergoren).

Die Kläranlage Zirl liegt im Inntal zehn Kilometer westlich von Innsbruck. Die Anlage wurde 1996 in Betrieb genommen und 2005 um eine eigene Schlammbehandlung erweitert. Die Anlage ist für $65.500 \mathrm{EW}_{60}$ ausgebaut. Der Fermenter verfügt über ein Volumen von $1350 \mathrm{~m}^{3}$. Seit dem Jahr 2007 werden an der Anlage mechanisch aufbereitete Bioabfälle mitvergoren. Bis 2010 wurden die Mengen an co-vergorenen Bioabfällen kontinuierlich gesteigert.

Die detaillierten Aufzeichnungen der ARA Strass über Arbeitszeiten, Reparatur- und Investitionskosten, $\mathrm{Zu}-$ und Ablauffrachten, Stromkosten etc. ermöglichten es, die Zusatzkosten der MAB-Co-Vergärung zu ermitteln. Betrachtet wurde dabei der Zeitraum 2004 bis 2007 (ohne Co-Fermentation) und 2008 bis 2014 (mit Co-Fermentation). Zur Ermittlung des durch die MABCo-Vergärung erzielten Methanmehrertrags wurden die Daten der ARA Zirl verwendet, da hier neben der Vergärung der Schlämme aus der Abwasserlinie in erster Linie MAB co-vergoren wurden, was einen Vergleich der Methanerträge mit und ohne Co-Vergärung von MAB ermöglicht. Fettabscheiderinhalte, de- 
ren Zusammensetzung und Methanertrag nur abgeschätzt werden kann, wurden an der ARA Zirl fallweise auch als Co-Substrate verwendet, jedoch in so geringen Mengen, dass sie in der folgenden Betrachtung nur einen geringen Einfluss auf das Ergebnis haben können.

\subsection{Kosten- und Erlöse der Co- Vergärung}

Im Rahmen dieser Untersuchung wurden Prozessschritte im Kläranlagenbetrieb analysiert, die bei der Co-Vergärung von $\mathrm{MAB}$ beteiligt sind und bei denen es durch die Co-Vergärung $\mathrm{zu}$ erhöhten Aufwendungen kommen könnte. In Abb. 1 sind die betrachteten Kostenpunkte bzw. Erlösoptionen dargestellt:

- Bioabfall (Annahme und Speicherung): Zur Annahme des MAB muss die Infrastruktur dafür an der Kläranlage errichtet werden. Weiterhin fallen zusätzliche Arbeitsstunden für die Übernahme des MAB an.

- Schlammstabilisierung: Der Bereich der Schlammstabilisierung behandelt neben den Schlämmen aus der Abwasserreinigung auch MAB. Mit Zugabe von $\mathrm{MAB}$ in den Faulturm der Kläranlage ist daher mit erhöhtem Aufwand in diesem Bereich zu rechnen.

- Abwasserreinigung: Bei der Co-Vergärung von Bioabfällen kommt es zu einer zusätzlichen Stickstoffrückbelastung in die Kläranlage.

- Methannutzung: Das zusätzlich aus der MAB-Co-Vergärung gewonnene
Biogas kann entweder mittels BHKW direkt verstromt werden und zur Abdeckung des Eigenstrombedarfs der Kläranlage genutzt oder in das Stromnetz eingespeist werden. Auch kann es weiter zu Erdgasqualität aufbereitet und in das Erdgasnetz eingespeist werden.

In dieser Arbeit wurden nur die Grenzkosten der MAB-Co-Vergärung berücksichtigt, d.h. die Kosten, die der Kläranlage zur Verarbeitung des MAB zusätzlich entstehen. Nicht berücksichtigt wurden die Investitionskosten der Kläranlage, z. B. für den Faulturm, für Entwässerungsaggregate etc. und die Aufbereitungskosten für den Bioabfall sowie die Transportkosten zur Kläranlage. Zudem wurden die Arbeitsstunden für zusätzliche Verwaltungsarbeiten, bedingt durch die MAB-Co-Vergärung nicht erhoben.

\subsection{Mechanisch aufbereiteter Bioabfall (MAB)}

Der MAB, welcher an den Kläranlagen Strass und Zirl verwertet wird, besteht im Wesentlichen aus Bioabfällen der Getrenntsammlung, überlagerten Lebensmitteln und Resten aus der Lebensmittelproduktion. Die Abfälle werden mit verschiedenen Aufbereitungsaggregaten, einer Trennmühle bzw. einer Abfallkolbenpresse, aufbereitet. Dabei werden die Abfälle auf eine Korngröße von etwa $10 \mathrm{~mm}$ zerkleinert. Über ein Sieb werden nach der Zerkleinerung v.a. Verpackungen wie z. B. Kunststofffolien entfernt, enthal-

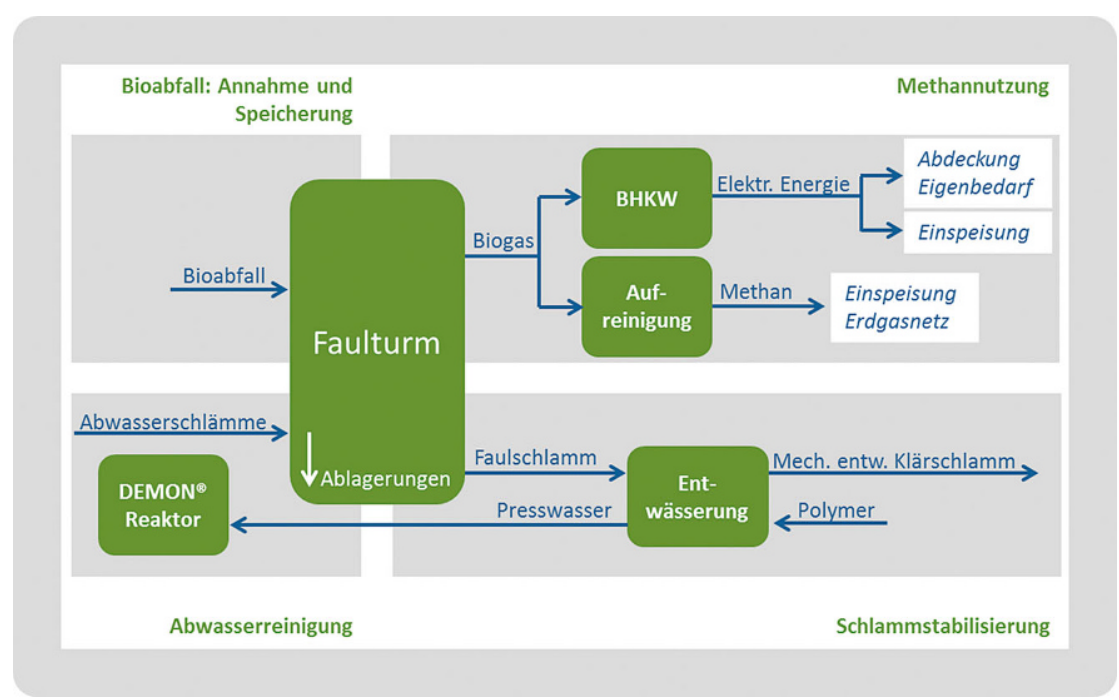

Abb. 1 Schematische Darstellung einer Kläranlage tene Inertstoffe werden zum Großteil zerkleinert und gelangen in das Co-Substrat. Durch Zugabe von Frisch- oder Brauchwasser wird der MAB verdünnt, bis dieser pumpfähig ist.

Die aufbereiteten Abfälle werden mit einem Trockenrückstands (TR)-Gehalt von etwa 12 bis $18 \%$ an der Kläranlage angeliefert. Den Berechnungen in dieser Arbeit wird ein mittlerer TR-Gehalt des MAB von $14 \%$ zugrunde gelegt. Die Bestimmung des Glühverlusts ergab einen mittleren organischen Trockenrückstands (oTR)-Gehalt von $83 \%$. Die an der Kläranlage Strass pro Jahr verarbeiteten Mengen an MAB lagen im Durchschnitt bei 3100 Tonnen/Jahr. An der ARA Zirl wurden zwischen 1500 und 3000 Tonnen MAB pro Jahr verarbeitet.

\subsection{Methanertrag bei der Co-Vergärung von MAB}

Durch die Zuführung von externen organischen Reststoffen soll ein Mehrertrag an Methan in den Faultürmen erzielt werden. Ziel ist eine möglichst weitgehende Umwandlung der organischen Fraktion in Methan, um einerseits die Energieausbeute zu maximieren und andererseits den Anfall an Reststoffen (Klärschlamm) zu minimieren. Um die Co-Fermentation gesamthaft bewerten zu können, muss der Methanmehrertrag, welcher durch die CoFermentation des MAB verursacht wird, bestimmt werden. An Kläranlagen, die Co-Vergärung betreiben, zeigte sich, dass Methanerträge gemessen wurden, die deutlich über den im Labor ermittelten Werten lagen. Die Differenz in der Methanmenge zwischen großtechnischen und den im Labor ermittelten Daten kann darauf zurückgeführt werden, dass die Schlämme aus der Abwasserbehandlung effektiver abgebaut werden und so zusätzlich Methan liefern (Maes et al. 2013). Mit einem vermehrten Schlammabbau durch die Co-Substratzugabe sind in Folge auch die Schlammmengen, die nach der Vergärung anfallen, reduziert. Obwohl ein erhöhter Methanertrag und ein zusätzlicher Schlammabbau großtechnisch beobachtet wurden, fehlen bis dato Laborversuche, die diesen Effekt nachbilden und Einflussfaktoren zeigen. Diesen zusätzlichen Methanabbau/Schlammabbau in Laborversuchen systematisch nachzuweisen, ist jedoch schwierig, da die im Labor ermittelten Werte zu Methanertrag und Reststoffanfall nur bedingt auf die großtechni- 


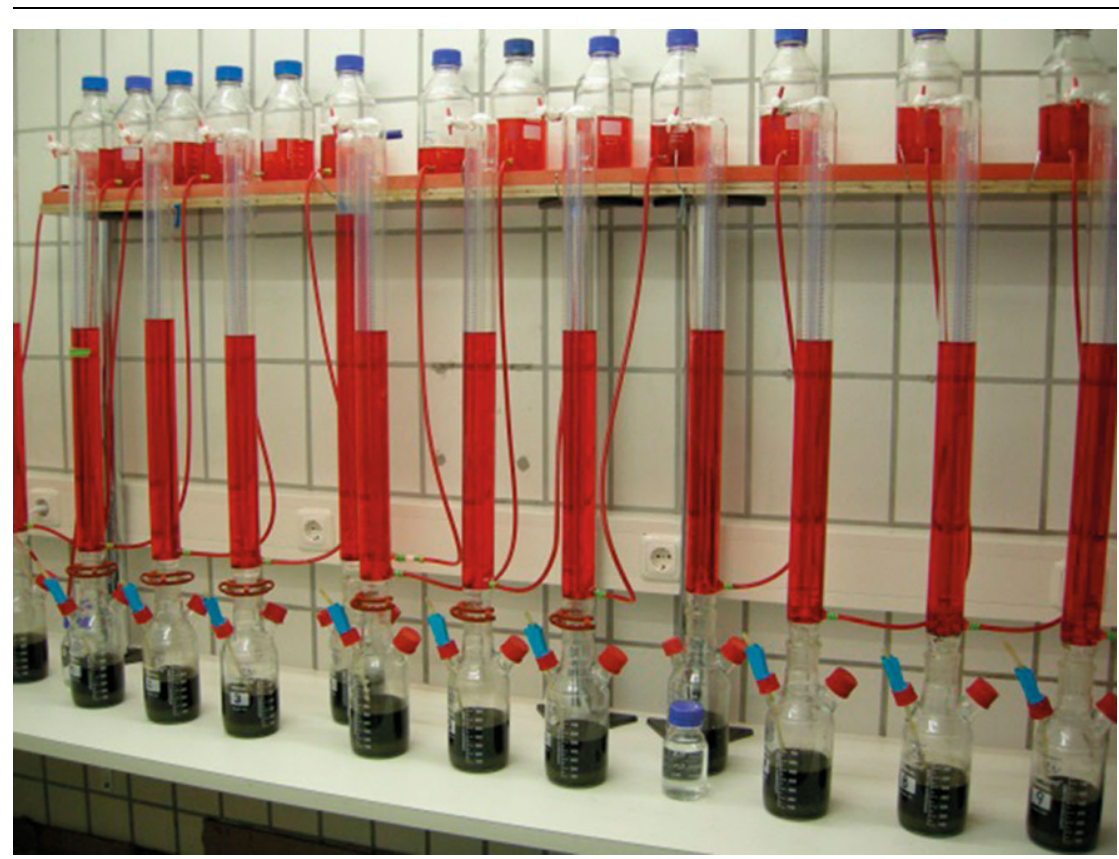

Abb. 2 Messapparatur zur Bestimmung der Methausbeute aus MAB

schen Bedingungen übertragbar sind. In dieser Arbeit werden ein möglicher großtechnischer Methanertrag aus MAB sowie der Methanertrag, welcher in Laborversuchen ermittelt wurde, dargestellt. Dieselbe Betrachtung wurde ebenfalls für den möglichen, zusätzlichen Abbau an mechanisch entwässerten Klärschlamm (MEK) durchgeführt.

\subsection{Berechnung des Methanertrags basierend auf Laborversuchen}

Um den Methanertrag von Co-Substraten $\mathrm{zu}$ bestimmen, können Labor-

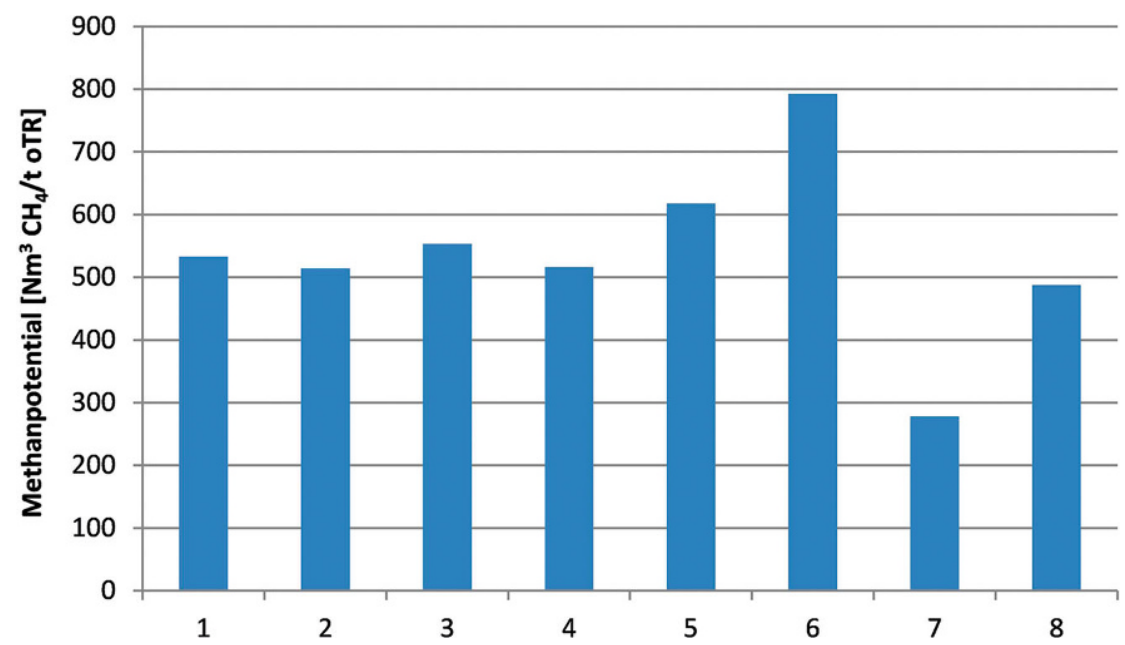

raum Februar bis April 2013 dargestellt. Die Methanausbeute ist auf den eingesetzten organischen Trockenrückstand (oTR) bezogen.

\subsubsection{Berechnung des Methanertrags basierend auf großtechnischen Daten}

Bei der Ermittlung des großtechnischen Methanertrages muss zwischen dem Methanertrag der Klärschlämme und dem Methanertrag der Co-Substrate differenziert werden. Eine Differenzierung in einer Praxisanlage ist dann möglich, wenn für die betreffende Anlage auch ein Bilanzzeitraum besteht, in dem nur Klärschlämme im Faulturm behandelt wurden. Der Methanertrag dieses Bilanzzeitraumes wird auf die CSB Zulauffracht der Kläranlage normiert (z. B. Nm ${ }^{3}$ Methan/t CSB). Umgerechnet auf EW $\mathrm{CSB}_{120}$ und Tag sollten Werte um die $12-14 \mathrm{Nl} \mathrm{CH}_{4} /$ $\mathrm{EWCSB}_{120} \times \mathrm{d}$ resultieren. Dies ist der klassische Methanertrag bei einstufigen Belebungsanlagen (mit Vorklärung) und Schlammfaulung (Merkblatt DWAM 363 2010; Merkblatt DWA-M 368 2014). Dieser Methanertrag ergibt sich, wenn man davon ausgeht, dass etwa $60 \%$ des CSB im Zulauf der Kläranlage über die Klärschlämme dem Faulturm zugeführt und davon ca. die Hälfte in Methan umgewandelt wird. In den Jahren, in denen der Schlammfaulung CoSubstrate zugeführt werden, kann nun der theoretische Methanertrag aus den Abwasserschlämmen aus der gemessenen CSB Zulauffracht der Kläranlage kalkuliert werden. Die Differenz zu dem tatsächlich in der Anlage gemessenen Gesamtgasertrag kann der CoFermentation zugeordnet werden. Im Jahr 2006 gab es an der ARA Zirl noch keine Co-Vergärung. Dieser Zeitraum diente zur Berechnung der Eckdaten der reinen Klärschlammvergärung. Für die Ermittlung des großtechnischen Methanertrages wurden die Prozessdaten der Kalenderjahre 2012, 2013 und 2014 herangezogen. Die angelieferten Mengen an MAB wurden immer verwogen und somit genau aufgezeichnet. Es wurde jeweils Jahresbilanzen der Kalenderjahre 2012, 2013 und 2014 erstellt. Der auf diese Weise errechnete mittlere Methanertrag beträgt $800 \mathrm{Nm}^{3} \quad \mathrm{CH}_{4} / \mathrm{t}$ oTR.

Abb. 3 Ergebnisse der Laborbatchversuche zur Bestimmung der Methausbeute aus MAB an der ARA Zirl 


\subsection{Eigennutzung des produzierten} Stroms bzw. Verkauf als Ökostrom

Eine mögliche Nutzung des produzierten Methans ist die Stromproduktion über ein Blockheizkraftwerk (BHKW) direkt an der Kläranlage. Für die Berechnungen der Strommenge wurde ein Wirkungsgrad des BHKW von $38 \%$ angenommen. Die Betriebskosten der BHKW an der Kläranlage AIZ betragen 2,5 Cent/kWh (inkl. Kapitalkosten). Der produzierte Strom kann zur Abdeckung des Eigenstrombedarfs an der Kläranlage genutzt werden. Im Falle dieser Eigennutzung wurde ein Strombezugspreis von $10 \mathrm{Cent} / \mathrm{kWh}$ als Erlös angesetzt. Da Strom aus Kläranlagen nicht als Ökostrom anerkannt und entsprechend gefördert wird, kann bei der Einspeisung des Stromes in das öffentliche Netz nur der Marktpreis für Strom angesetzt werden. Im Rahmen dieser Betrachtung wurde ein Erlös von 3,5 Cent/kWh verwendet und den Produktionskosten gegenübergestellt.

\subsection{Verkauf als Biogas}

Eine weitere Verwertungsoption ist die Abgabe des Biogases an einen Gasaufbereiter. Für den Raum Innsbruck lag ein Angebot von 1,95 Cent/kWh Rohbiogas vor.

\subsection{Anfall an mechanisch entwässertem Klärschlamm}

Die Zugabe von MAB in den Kläranlagenfaulturm beeinflusst die Menge an MEK, die nach der Vergärung entwässert und anschließend entsorgt werden muss. Unter der Annahme, dass sich durch den MAB kein verändertes Entwässerungsverhalten ergibt, was in weiteren Betrachtungen noch $\mathrm{zu}$ untersuchen wäre, sind der Methanertrag und der Anfall an MEK voneinander abhängige Größen, da die zugeführte organische Substanz entweder zu Methan umgewandelt wird oder als Biomasse den Faulturm nach der Vergärung wieder verlässt. Es wird angenommen, dass der mineralische Anteil in den Co-Substraten während der Vergärung keine Veränderung erfährt und sich in Folge unverändert im Gärrest wiederfindet.

\subsubsection{MEK-Anfall bei Methanausbeute nach Laborversuchen}

Bei einer mittleren Labormethanausbeute von $500 \mathrm{Nm}^{3} \mathrm{CH}_{4} / \mathrm{t}$ oTR und ei- nem Methangehalt im Biogas von $62 \%$ werden $806 \mathrm{Nm}^{3}$ Biogas/t oTR produziert. Der Abbaugrad der Biomasse errechnet sich wie folgt:

$$
\text { Abbaugrad }(\%)=\frac{\text { Biogas }}{V_{m}} * M_{C} * f(1)
$$

Gl. 1 Berechnung des Abbaugrads der Biomasse.

Vm Molares Volumen (22,4l/mol)

Mc Molare Masse Kohlenstoff $(12 \mathrm{~g} / \mathrm{mol})$

f Kohlenstoffgehalt des abgebauten oTR; Faktor: 1,9 (Reinhold 2013)

Diese Berechnung ergab einen $\mathrm{Ab}$ baugrad von $82 \%$. Unter der Annahme, dass die mineralische Trockensubstanz unverändert das System passiert und die organische Trockensubstanz zu $82 \%$ abgebaut wird, kann insgesamt ein MEK-Anfall von $150 \mathrm{~kg} / \mathrm{t}$ MAB berechnet werden. Der TR-Gehalt des entwässerten Klärschlamms lag dabei im Durchschnitt bei $29 \%$.

\subsubsection{MEK-Anfall bei großtechnischer Methanausbeute}

Wird eine großtechnische Methanausbeute von $800 \mathrm{Nm}^{3} \mathrm{CH}_{4} / \mathrm{t}$ oTR angenommen, bedeutet das bei einem Methangehalt im Biogas von $62 \%$, dass pro Tonne oTR des MAB $1290 \mathrm{Nm}^{3}$ Biogas produziert werden. Daraus errechnet sich ein Abbaugrad der Biomasse von $130 \%$ (vgl. Gl. 1), d. h. neben dem MAB wird auch zusätzliche organische Substanz aus den Abwasserschlämmen abgebaut. Damit kommt es unter Berücksichtigung des mineralischen Anteils im MAB bei der Co-Vergärung rechnerisch zu keinem zusätzlichen MEK-Anfall.

\subsection{Flockungshilfsmittel}

Aus dem Faulturm abgeführter Klärschlamm wird zur Volumensreduktion entwässert. Der MEK wird entsorgt, die abgepresste Flüssigkeit in den Prozess der Abwasserbehandlung rückgeführt. Vor der Entwässerung werden dem Klärschlamm Flockungshilfsmittel zugesetzt, um die Entwässerbarkeit des Klärschlammes zu erhöhen. Mit steigender Klärschlammmenge steigen auch die benötigten Mengen an Flockungshilfsmitteln. Wie unter 2.7.1 beschrieben, wurde der MEK-Anfall durch die Zugabe von MAB bei einem Methanertrag lt. Laborversuchen berechnet. Der Anteil des MEK aus MAB am Gesamt-MEK-Anfall kann berech- net werden. Wird dieser Anteil auf die jährlich benötigte Menge an Flockungshilfsmittel umgelegt, kann auf die aufgrund der MAB-Co-Vergärung zusätzlich benötigte Menge an Flockungshilfsmittel rückgeschlossen werden. Unter der Annahme einer großtechnischen (erhöhten) Methanausbeute und damit keines Schlammmehranfalls wird rein theoretisch auch kein zusätzliches Flockungshilfsmittel benötigt.

\subsection{Stromkosten zur Stickstoffentfernung aus Presswasser}

Der Stickstoff, der über den MAB in den Faulturm der Kläranlage gebracht wird, wird bei der Co-Vergärung zu löslichem Ammonium umgewandelt und gelangt nach der Entwässerung des Faulschlamms über das Presswasser wieder in die Abwasserreinigung der Kläranlage. Das vermehrte Einbringen von Stickstoff führt an der Kläranlage zu erhöhten Aufwendungen in der Abwasserreinigung. An der ARA Strass wird der Stickstoff im Schlammwasser nach der Entwässerung des Klärschlamms in einem sog. DEMON ${ }^{\circledR}$-Reaktor entfernt. In belüfteten und unbelüfteten Phasen dieses Prozesses wird zuerst ein Teil des vorhandenen Ammoniums zu Nitrit oxidiert und dann im nächsten Schritt das restliche Ammonium anaerob mit dem gebildeten Nitrit zu $\mathrm{N}_{2}$ oxidiert. Der zusätzliche Strombedarf, den der $\mathrm{DEMON}^{\circledR}$-Reaktor aufgrund des $\mathrm{zu}$ sätzlichen Stickstoffs aus der MAB-CoVergärung benötigt, wird mithilfe des Bezugsjahres 2006 berechnet, in dem an der ARA Strass noch kein MAB verwertet wurde (vgl. Abb. 4).

Der Energieaufwand für den zusätzlich $\mathrm{zu}$ behandelnden Stickstoff wurde berechnet, indem im Vergleichszeitraum ohne Co-Vergärung, der Anteil der Stickstoffrückbelastung an der Stickstoffzulauffracht berechnet wurde. Mithilfe dieses Anteils kann die Stickstoffrückbelastung, die dem Abwasser zuzuschreiben ist, im Vergleichszeitraum mit MAB-Co-Vergärung berechnet werden. In den Jahren mit MABCo-Vergärung kann von der Gesamtstickstoffrückbelastung der Anteil des Abwassers abgezogen und die Rückbelastung aus dem MAB berechnet werden. Der Prozentsatz der Rückbelastung aus MAB an der Gesamtrückbelastung wird mit den DEMON ${ }^{\circledR}$-Stromkosten multipliziert, um die Stromkosten für die Presswasserbehandlung des MABs 


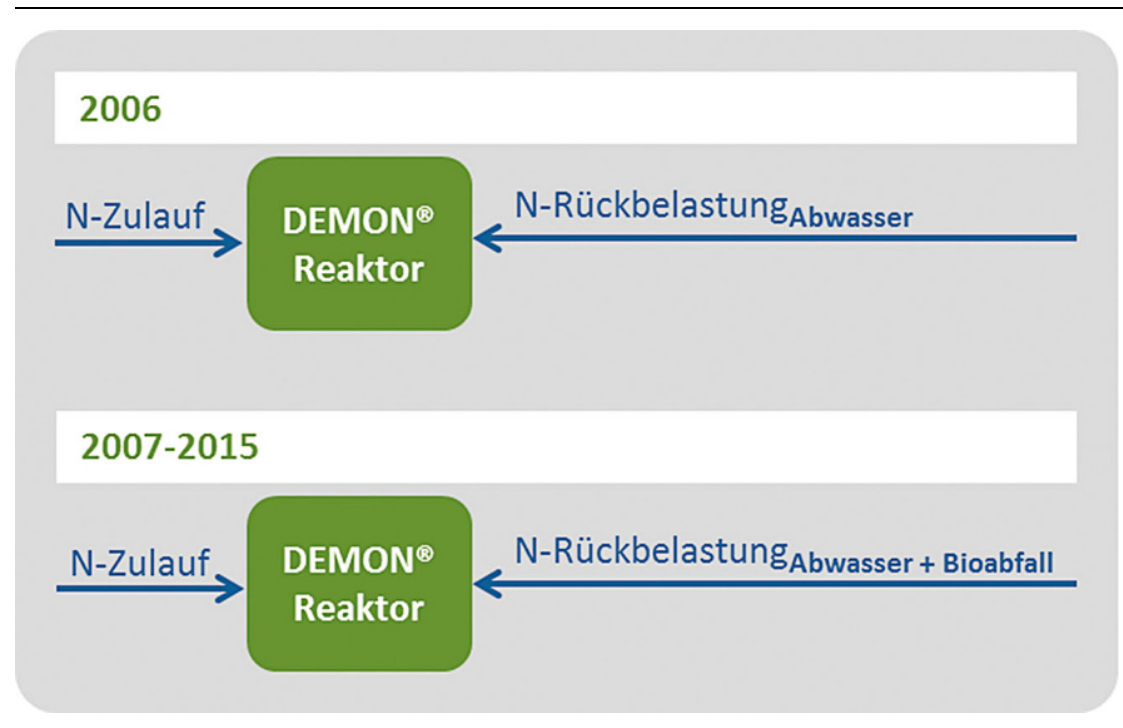

Abb. 4 BerechnungsschemaStromkostenDEMON ${ }^{\circledR}$ ohnebzw. mitCo-Vergärung von MAB

zu erhalten. Die Kosten für den Strombezug zum Betrieb des DEMON ${ }^{\circledR}$-Reaktors wurden mit $10 \mathrm{Cent} / \mathrm{kWh}$ angesetzt.

2.11 Investitions-, Reparaturkosten und zusätzliche Arbeitszeit

Im Zuge der MAB-Co-Vergärung wurde an der Kläranlage in Strass eine Übernahmestation für die Abfälle errichtet. Weiterhin wurden zusätzliche Pumpen sowie eine Radarmessung für den MABTank installiert. Diese Investitionskosten wurden auf einen Abschreibungszeitraum von 15 Jahren gerechnet.

Reparaturkosten wurden für Anlagenteile erhoben, die an der Verarbeitung des MAB beteiligt sind bzw. damit in Kontakt kommen. Dies umfasst folgende Komponenten: Faulturm inkl. Beschickung und Umwälzung, Voreindicker, Mischeindicker (vorwie-

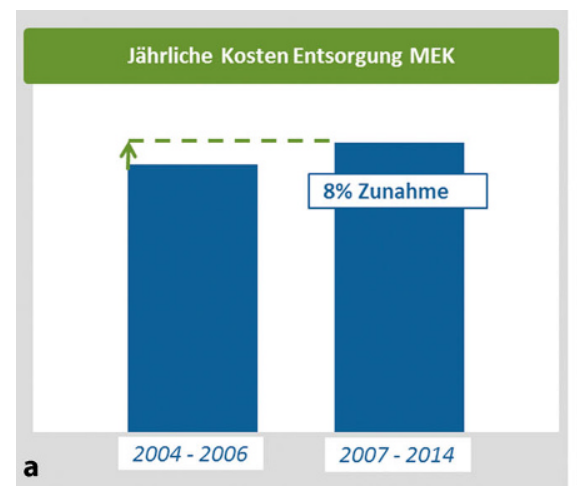

gend Rührwerke), Co-Substratbehälter (inkl. Pumpen für Übernahme), Macerator (Faulturm-Beschickung), Nacheindicker bzw. Stapelbehälter. Für die genannten Komponenten wurden die jährlichen Reparaturkosten für die Jahre ohne bzw. mit MAB-Co-Vergärung erhoben und die Reparaturkosten berechnet, die der MAB-Co-Vergärung zuzuschreiben sind.

Mithilfe der Stundenaufzeichnungen für die Bereiche Schlammstabilisierung (laufende Instandhaltung und Reparatur/Umbauten) und Co-Substrat konnten die mittleren jährlichen Arbeitsstunden für die betrachteten Jahre ohne bzw. mit MAB-Co-Vergärung berechnet und einander gegenübergestellt werden. Es wurde mit einem Stundensatz von $30 €$ gerechnet.

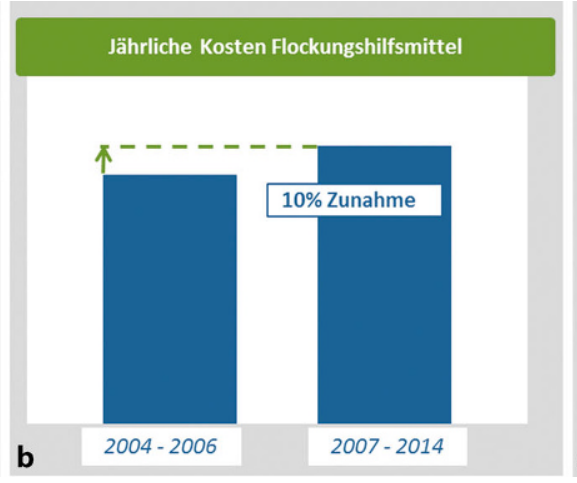

\subsection{Kosten Faulturmräumung}

Von April 2014 bis April 2017 wurden im Rahmen des alpS-Forschungsprojekts „ARAFerm“ verschiedenste Aspekte der Co-Vergärung von MAB an Kläranlagen gemeinsam mit ForschungspartnerInnen der Universität Innsbruck sowie Unternehmen und Kläranlagen aus Nord- und Südtirol untersucht. Ein Inhaltspunkt dieses Projekt war das Thema Störstoffe im MAB und deren Auswirkungen auf die Kläranlage. Dazu wurde eine Methode entwickelt, um Störstoffe im MAB zu bestimmen (Jank et al. 2016). Störstoffe im MAB sowie Störstoffe, die über die Abwasserlinie in den Faulturm gelangen, wurden quantifiziert. Das Ausmaß von Ablagerungen in Faultürmen von Kläranlagen mit MAB-Co-Vergärung wurde mithilfe einer Lithium-Tracer-Methode bestimmt. Im Zuge von Faulturmräumungen wurden Proben von Ablagerungen genommen, um die Art und Beschaffenheit dieser Sedimente bestimmen zu können. Mit diesen Informationen konnte die Menge an Ablagerungen, die der Zugabe von MAB zuzuschreiben sind, ermittelt werden. Grundlage der Kostenerhebung einer Entfernung von Störstoffsedimenten aus dem Faulturm waren ein Angebot von Faulturmtauchern sowie eine Kostenaufstellung für eine Faulturmräumung, die von der Kläranlage selbst durchgeführt wurde (Jilg 2012).

\section{Ergebnisse und Diskussion}

Unter der Annahme eines Methanertrags von $500 \mathrm{Nm}^{3} \mathrm{CH}_{4} / \mathrm{t}$ oTR aus dem Bioabfall ergeben sich Zusatzkosten aufgrund der MAB-Co-Vergärung für die Entsorgung des MEK, für das Flockungshilfsmittel zur Entwässerung

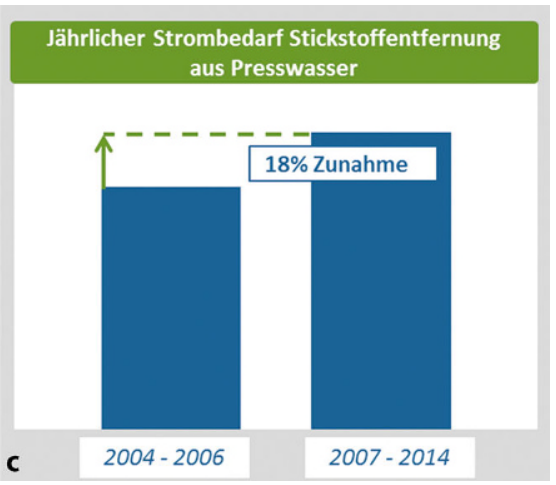

Abb. 5 Zunahme derjährlichen Kosten für die Entsorgung des MEK, für die Flockungshilfsmittel und den Strombedarf zur Stickstoffentfernung aus dem Presswasser im Zeitraum ohne und mit MAB-Co-Vergärung 


\section{Jährliche Reparaturkosten Anlagenkomponenten, die MAB mitverarbeiten}

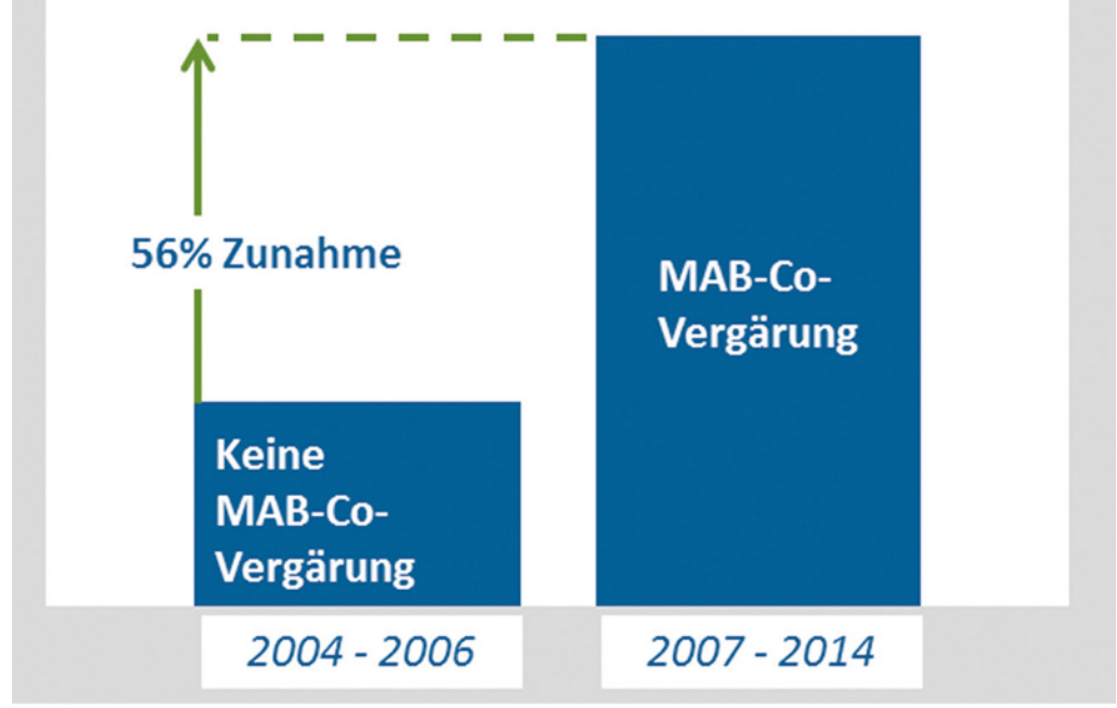

Abb. 6 Vergleich der jährlichen Reparaturkosten für Faulturm (inkl. Beschickung), Mischeindicker (vorwiegend Rührwerk) etc. ohne und mit MAB-Co-Vergärung

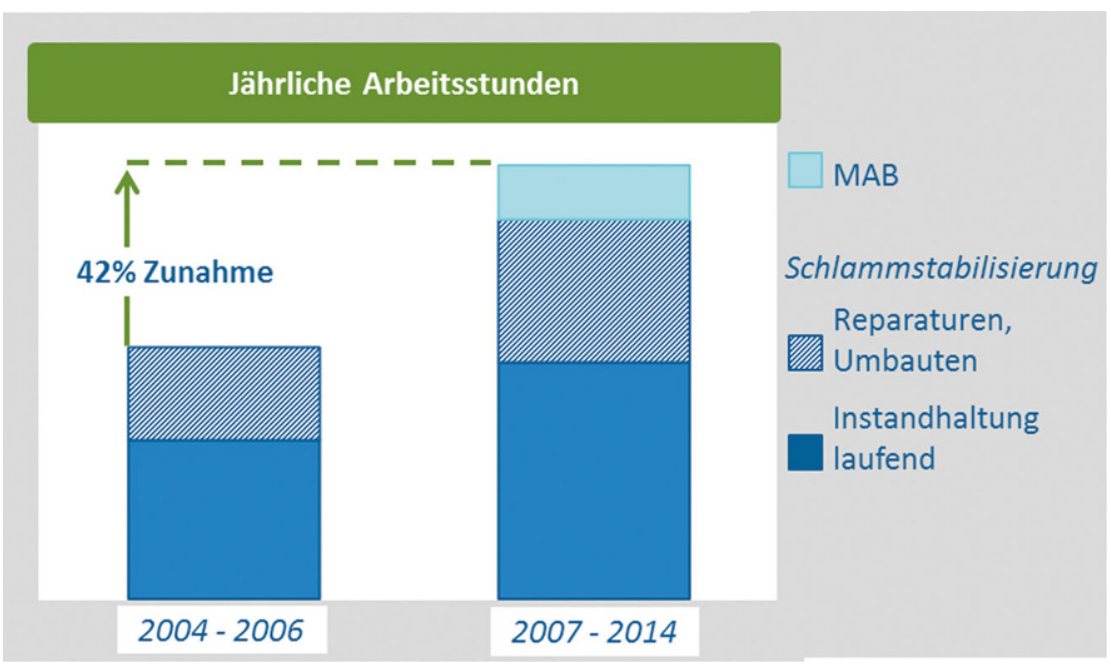

Abb. 7 Arbeitsstunden ohne und mit MAB-Co-Vergärung

und für die Behandlung des Presswassers. Die Steigerung der Kosten für die einzelnen Bereiche sind in Abb. 5 dargestellt. Die Zunahme der Kosten für die Entsorgung des MEK und die zusätzlich benötigten Flockungshilfsmittel lagen bei acht Prozent bzw. zehn Prozent. Der jährliche Strombedarf nahm durch die MAB-Co-Vergärung um $18 \%$ zu.

Beim Vergleich der Reparaturkosten (Materialkosten ohne Arbeitszeit) in den Jahren ohne und mit MAB-CoVergärung zeigte sich eine deutliche $\mathrm{Zu}$ -
3.1 Kosten und Erlöse für die Kläranlage bei der Verarbeitung einer Tonne MAB

In Abb. 8 sind die Erlöse, die durch Einspeisung des aus dem Co-Substrat produzierten Stroms, durch Abdeckung des Eigenstrombedarfs bzw. durch Abgabe des Biogases erzielt werden können, den zusätzlichen Aufwendungen für die Verarbeitung des Co-Substrats gegenübergestellt. Weiterhin sind die im Laborversuch und in der Praxis ermittelten Gaserträge aufgeführt. Die höchsten Erlöse werden erzielt, wenn der durch die Co-Vergärung von $\mathrm{MAB}$ produzierte Strom zur Abdeckung des Eigenbedarfs der Kläranlage genutzt wird. Bei Bezugskosten des Stroms von 10 Cent/kWh kann durch die Eigenabdeckung des Stroms unter Annahme des Laborgasertrages ein Erlös von $16 € / t$ MAB erzielt werden, bei Ansatz des höheren Gasertrages in der Praxis steigt der Erlös auf $26 € / t$. Deutlich geringer fällt der Erlös aus, wenn der Strom ins Stromnetz eingespeist wird. In diesem Fall kann ein Erlös von maximal $3 € / t$ MAB erzielt werden. Die BHKW Betriebskosten (2,5 Cent/kWh) wurden dabei bereits abgezogen. Bei der Abgabe des Biogases zur Aufbereitung ergibt sich ein Erlös von 11 (Laborgasertrag) bzw. $18 € / \mathrm{t}$ (großtechnischer Gasertrag).

Unter Annahme eines Methanertrags wie in Laborversuchen ermittelt, können nur bei einer Eigennutzung des produzierten Stroms die anfallenden Kosten in etwa gedeckt werden. Dem Erlös von $16 € / \mathrm{t}$ MAB stehen Kosten von $18 € / t$ MAB gegenüber. Unter diesen Bedingungen kann der $\mathrm{MAB}$ von der Kläranlage zum Nulltarif angenommen werden. Über das Jahresmittel wird die Kläranlage die zusätzlichen Kosten, die durch Schlammentsorgung, Reparaturen etc. entstehen, in etwa durch geringere Stromkosten ausgleichen können. Wird Strom ins Netz eingespeist, bzw. das Biogas abgegeben, müsste die Kläranlage zwischen 7 bis $16 € / t$ MAB für die Übernahme des MAB verlangen, um die Kosten der Verarbeitung des MAB decken zu können.

Den größten Kostenpunkt mit über $9 € / t$ MAB stellen die Entwässerung und Entsorgung des mechanisch entwässerten Klärschlammes nach der Vergärung dar. Dieser Kostenpunkt ist abhängig von der Entwässerungsleistung der eingesetzten Aggregate und den 


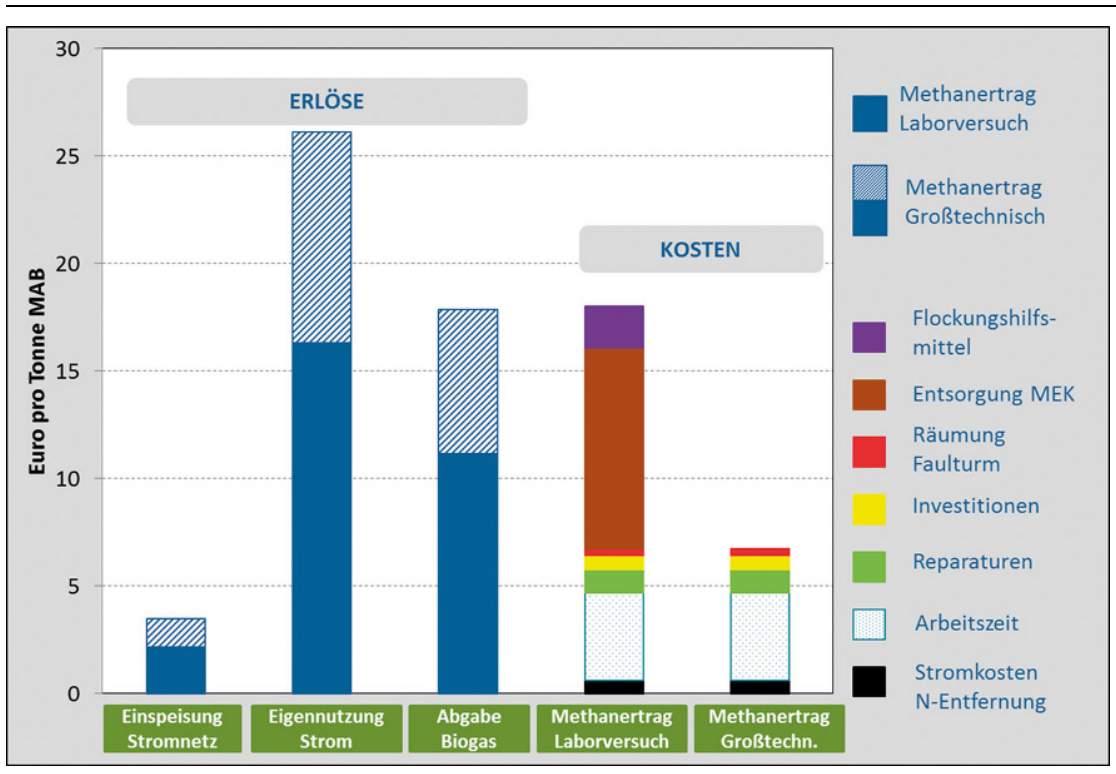

Abb. 8 Erlös und Kosten der MAB-Co-Vergärung auf Kläranlagen unter der Annahme unterschiedlicher Methanerträge und Abgabe- bzw. Verwertungsoptionen des gebildeten Methans

Tarifen zu welchen der Klärschlamm abgegeben werden kann. In den Jahren 2004 bis 2015 erhöhten sich die Entsorgungskosten des Klärschlammes von $47 € / \mathrm{t}$ auf $71 € / \mathrm{t}$. In Anbetracht dieses Trends ist auch für die kommenden Jahre mit steigenden Kosten für die Klärschlammentsorgung $\mathrm{zu}$ rechnen. Die Kosten des Flockungshilfsmittels belaufen sich auf knapp $2 € / \mathrm{t}$ MAB.

Der zweitgrößte Kostenpunkt mit $4 € / \mathrm{t}$ MAB ist die zusätzliche Arbeitszeit, die bei der Verarbeitung von MAB anfallen. Dies sind im Wesentlichen die Bereiche Schlammstabilisierung, laufende Reparaturen und Handling MAB.

Ein Punkt, der im Vergleich zu den Gesamtkosten eine eher untergeordnete Rolle spielt, sind die Stromkosten $(0,6 € / t \mathrm{MAB})$ für die Entfernung des zusätzlichen Stickstoffs im Presswasser, welches im Hauptstrom der Kläranlage gereinigt werden muss. An der Anlage in Strass wird der Ammoniumstickstoff, wie in 2.1 beschrieben, in einem DEMON ${ }^{\circledR}$-Reaktor entfernt. Dieses Verfahren entfernt Stickstoffe effizient und stromsparend. An der Kläranlage in Zirl wird der Stickstoff im Belebungsbecken der Abwassereinigung entfernt. Dort ergab die Erhebung der Kosten für die zusätzlich notwendige Stickstoffentfernung durch die MAB-Co-Vergärung in etwa das Doppelte der in Strass berechneten Kosten.

Die Kosten für die Abschreibung der erforderlichen zusätzlichen Inves- titionen zur MAB-Co-Vergärung belaufen sich auf $0,6 € / t$ MAB. Die Kosten für erhöhte Reparaturen, mutmaßlich hervorgerufen durch die Störstoffe im MAB, sowie die zusätzlichen Aufwendungen für Räumungen des Faulturms aufgrund von Störstoffsedimentation belaufen sich auf $1,4 € / t$ MAB. Damit sind die Kosten, die auf mechanische Beanspruchung durch Störstoffe und Sedimentation von Störstoffen zurückzuführen sind, im Vergleich zu den Gesamtkosten der MAB-Co-Vergärung vergleichsweise gering.

Wird bei dieser Berechnung ein Methanertrag angesetzt, wie dieser in Untersuchungen zum großtechnischen Methanertrag ermittelt wurde, können Erlöse bis zu $26 € / \mathrm{t}$ MAB erreicht werden, wenn der Strom an der Anlage direkt genutzt wird. Unter der Annahme eines großtechnischen Methanertrags können die Verarbeitungskosten des MAB durch Eigennutzung des Stroms und Abgabe des Biogases klar gedeckt werden. Durch den höheren Gasertrag ergibt sich zwangsläufig auch ein höherer Abbau der organischen Substanz und damit verbunden ein niedrigerer Klärschlammanfall. Bei den angesetzten Biogasmengen aus den großtechnischen Daten ergibt sich rechnerisch kein zusätzlicher Klärschlamm. Damit entfallen auch die entsprechenden Klärschlammentsorgungskosten und auch die Kosten für Flockungshilfsmittel. Unter diesen Bedingungen stehen
Kosten von $6,7 € / \mathrm{t}$ MAB Erlösen von bis $\mathrm{zu} 26 € / \mathrm{t}$ MAB gegenüber. Nur die Stromeinspeisung bleibt auch bei der Annahme eines großtechnischen Methanertrags eine für die Kläranlage unwirtschaftliche Option. Da an den betrachteten Anlagen zwar ein entsprechend höherer Methanertrag als im Laborversuch gemessen wurde, die Daten aber gewisse Unsicherheiten aufweisen, um diesen Effekt mit Sicherheit belegen zu können, stellt der geringe Methanertrag aus den Laborversuchen für eine Kalkulation der Wirtschaftlichkeit der MAB-Co-Vergärung einen sicheren Ansatz dar. Wird in weiteren Untersuchungen ein erhöhter Methanertrag bei der MAB-Co-Vergärung im Vergleich $\mathrm{zu}$ den Laborversuchen und damit einhergehend ein erhöhter Schlammabbau nachgewiesen, kann die Kläranlage die Wirtschaftlichkeit einer MAB-Co-Vergärung aufgrundlage dieser Daten kalkulieren.

\section{Fazit}

Bei der Entscheidung zur Co-Vergärung von $\mathrm{MAB}$ an Kläranlagen sollten im Wesentlichen die möglichen Verwertungsoptionen der durch die Co-Vergärung zusätzlich gewonnenen Energie betrachtet werden. Unter den aktuellen Bedingungen, dass die in den Faultürmen von Kläranlagen erzeugte erneuerbare Energie in Österreich nicht als Ökostrom anerkannt ist und daher keine entsprechende Vergütung erhält, stellt die Eigennutzung der aus dem Methan produzierten elektrischen Energie für die Kläranlage die finanziell attraktivste Option dar. Die Abgabe der produzierten elektrischen Energie in das öffentliche Stromnetz ist aufgrund der niedrigen Einspeisvergütung nicht rentabel. Um hier eine höhere Einspeisevergütung zu erzielen, wäre die Produktion von Spitzenstrom, also die für das Stromnetz bedarfsgerechte Bereitstellung von elektrischer Energie, eine Möglichkeit. Um dies umzusetzen sind jedoch Speicher für den MAB, für das produzierte Biogas und entsprechend ausgelegte BHKWs notwendig.

Die Wirtschaftlichkeit der MAB-CoVergärung an der Kläranlage ist ganz entscheidend von der aus dem Abfall produzierten Methanmenge abhängig. Zum einen wegen der produzierten Methanmenge selbst, zum anderen sinkt mit der Umwandlung des Kohlenstoffes in Methan die Menge an Klärschlamm, welcher von der Kläranlage entsorgt 
werden muss. Da die Entsorgungskosten des Klärschlammes aus MAB den mit Abstand größten Kostenpunkt bei der Co-Vergärung darstellen, ist für ein möglichst wirtschaftliches Betreiben der MAB-Co-Vergärung eine hohe Umwandlung des zugeführten Kohlenstoffes in Methan entscheidend. Im Zuge der Auswertung der Labor-und großtechnischen Daten mehrerer Co-Vergärungsanlagen hat sich gezeigt, dass in diesem Bereich sehr große Schwankungsbreiten vorhanden sind. Dies hat sicherlich mit der Qualität der MAB zu tun, die in der Praxis großen Schwankungen unterworfen ist. Besteht der MAB vor allem aus reinen Küchenabfällen (mit hohem Anteil an leicht abbaubaren Komponenten) so sind in aller Regel hohe Methanerträge und niedrige Reststoffmengen zu erwarten.

In der Diskussion um die Mitverarbeitung von organischen Abfällen in den Faultürmen von Kläranlagen werden erhöhte Aufwendungen durch die in den Co-Substraten enthaltenen Störstoffe ins Feld geführt. Kleine Steine, Glasteilchen oder auch Sand können z. B. bei Pumpen zu vermehrtem Verschleiß führen, was zu zusätzlichen Reparatur- und Arbeitszeiten führt. Die Erfahrungen auf der ARA Strass zeigen jedoch, dass die durch die Störstoffe hervorgerufenen Kosten in den $\mathrm{Ge}$ samtkosten für die Vergärung eine eher untergeordnete Rolle spielen.

Dennoch trägt ein sauberes Substrat zur Gesamtwirtschaftlichkeit der Co-Vergärung bei und Konzepte zur Verbesserung der MAB-Qualität durch eine effiziente Störstoffabtrennung, wie z. B. Hydrozyklone können hier zu einer weiteren Optimierung beitragen.

Danksagung Diese Arbeit wurde im Rahmen des alpS-Projekts EneRAlp II Teil B „ARAFerm“ durchgeführt. alpS wird im Rahmen von COMET - Competence Centers for Excellent Technologies durch BMVIT, BMWFW, Land Tirol und Land Vorarlberg gefördert. Das Programm COMET wird durch die FFG abgewickelt. Vielen Dank den Kläranlagen Strass und Zirl für die Bereitstellung von Betriebsdaten und für die Unterstützung beim Verfassen dieser Arbeit.

Open access funding provided by University of Innsbruck and Medical University of Innsbruck.

Open Access Dieser Artikel wird unter der Creative Commons Namensnennung 4.0 International Lizenz (http:// creativecommons.org/licenses/by/4. 0/deed.de) veröffentlicht, welche die Nutzung, Vervielfältigung, Bearbeitung, Verbreitung und Wiedergabe in jeglichem Medium und Format erlaubt, sofern $\mathrm{Sie}$ den/die ursprünglichen $\mathrm{Au}$ tor(en) und die Quelle ordnungsgemäß nennen, einen Link zur Creative Commons Lizenz beifügen und angeben, ob Änderungen vorgenommen wurden.

\section{Literatur}

Aichinger, P, Kuprian, M., Probst, M. Insam, H., Ebner, C. (2015): Demand-driven energy supply from stored biowaste for biomethanisation. Bioresour Technol 194, 389-393.

Bayo, J., López-Castellanos, J., Martínez-García, R., Alcolea, A., Lardín, C. (2015): Hydrocyclone as a cleaning device for anaerobic sludge digesters in a wastewater treatment plant. Journal of Cleaner Production 87, 550-557.

Dai, X., Duan, N., Dong, B., Dai, L. (2013): Highsolids anaerobic co-digestion of sewage sludge and food waste in comparison with mono digestions: stability and performance. Waste Manag $33,308-316$.

Ebner, C. (2013): Co-Fermentation von biogenen Abfällen. KA Betriebs-Info 3/13, 2119-2123. Iacovidou, E., Ohandja, D.G., Voulvoulis, N. (2012): Food waste co-digestion with sewage sludge - realising its potential in the UK. J Environ Manage 112, 267-274.

Jank, A., Muller, W., Waldhuber, S., Gerke, F. Ebner, C., Bockreis, A. (2016): Impurities in pretreated biowaste for co-digestion: A determination approach. Waste Manag 52, 96-103.

Jilg, M. (2012): Alte Zöpfe abgeschnitten - Komplettentleerung der Faulbehälter hielt manche Überraschung bereit. KA Betriebs-Info 2/2012, 1912-1914.

Kim, H.W., Nam, J.Y., Shin, H.S. (2011): A comparison study on the high-rate co-digestion of sewage sludge and food waste using a temperature-phased anaerobic sequencing batch reactor system. Bioresour Technol 102, 7272-7279.
Krupp, M., Schubert, J., Widmann, R. (2005): Feasibility study for co-digestion of sewage sludge with OFMSW on two wastewater treatment plants in Germany. Waste Management 25, 393-399.

Lastella, G., Testa, C., Cornacchia, G., Notornicola, M., Voltasio, F., Sharma, V.K. (2002): Anaerobic digestion of semi solid organic waste: biogas production and its purification. Energy Conversion and Management 43, 63-75.

Maes, L., Weemaes, M., Hellinck, N., De Gueldre, G., Van De Steene, B. (2013): Co-digestion of glycerine and sewage sludge to optimise green electricity production. Water Sci Technol 67, 2863-2867.

Merkblatt DWA-M 363 (2010): Herkunft, Aufbereitung und Verwertung von Biogasen. Deutsche Vereinigung für Wasserwirtschaft, Abwasser und Abfall e.V.

Merkblatt DWA-M 368 (2014): Biologische Stabilisierung von Klärschlamm. Deutsche Vereinigung für Wasserwirtschaft, Abwasser und Abfal e.V.

Montusiewicz, A., Lebiocka, M. (2011): Co-digestion of intermediate landfill leachate and sewage sludge as a method of leachate utilization. Bioresour Technol 102, 2563-2571.

Novarino, D., Zanetti, M.C. (2012): Anaerobic digestion of extruded OFMSW. Bioresour Technol 104, 44-50.

Reinhold, G. (2013): Einfluss der Fermentation auf den Abbau der Inhaltsstoffe, Fachtagung "Pflanzenbauliche Verwertung von Garruck- ständen aus Biogasanlagen“. Umweltforum Auferstehungskirche Berlin.

Romero-Güiza, M.S., Peces, M., Astals, S., Benavent, J., Valls, J., Mata-Alvarez, J. (2014) Implementation of a prototypal optical sorter as core of the new pre-treatment configuration of a mechanical-biological treatment plant treating OFMSW through anaerobic digestion. Applied Energy 135, 63-70.

Schneider, I., Müller, W., Bockreis, A. (2015): Optimierte Erfassung und Lagerung von Speiseresten aus Kantinen und Gastronomiebetrieben, in: Bockreis, A., Faulstich, M., Flamme, S., Kranert, M., Mocker, M., Nelles, M., Quicker, P, Rettenberger, G, Rotter, V.S. (Eds.), 5. Wissenschaftskongress Abfall- und Ressourcenwirtschaft. innsbruck university press (IUP), Innsbruck, pp. 57-60.

Shen, Y., Linville, J.L., Urgun-Demirtas, M. Mintz, M.M., Snyder, S.W. (2015): An overview of biogas production and utilization at full-scale wastewater treatment plants (WWTPs) in the United States: Challenges and opportunities towards energy-neutral WWTPs. Renewable and Sustainable Energy Reviews 50, 346-362.

Silvestre, G., Rodriguez-Abalde, A., Fernandez, B., Flotats, X., Bonmati, A. (2011): Biomass adaptation over anaerobic co-digestion of sewage sludge and trapped grease waste. Bioresour Technol 102, 6830-6836.

VDI 4630 (2014): Vergärung organischer Stoffe. Beuth Verlag, Berlin. 